\title{
The Ultimate Anti-Seismic System
}

\author{
N. Lymperis Ioannis \\ Ios Island, Cyclades ZIP Code 84001, Greece \\ Email: lymperis ios@yahoo.com
}

Received 26 July 2015; accepted 21 September 2015; published 24 September 2015

Copyright (C) 2015 by author and Scientific Research Publishing Inc.

This work is licensed under the Creative Commons Attribution International License (CC BY).

http://creativecommons.org/licenses/by/4.0/

c) (i) Open Access

\begin{abstract}
In general, of construction projects, utility anti-seismic technologies, such as hydraulic tie rod or tie rod structural vibration control system, assess efficiency and safety in node structures from earthquakes risk. According the building construction framework, we exanimate, with experiments, the mechanism of reaction and a patent idea of anti-seismic system with potential conclusions, through using different methods, such as of horizontal seismic insulation, design with elongated rigid columns and design columns without ductility. We provide that we made strong foundation in earthquakes, solar-wind and utility power structural systems and tornados effects in lightweight constructions. We suggest that may fix to existing or under design construction like bridges.
\end{abstract}

\section{Keywords}

Vibration, Control-System, Anti-Seismic, Earthquakes, Construction

\section{Introduction}

The decade's earthquakes around the world have put first priority of the major social and economic issue of the seismic behavior and the overall seismic protection of structures against earthquake disaster. Due to the necessity of containing the effects of the seismic we have developed various methods to optimize the response of structure motions.

According to modern standard, the seismic design of building is based on the capacity design and ductility requirements. The inevitable inelastic behavior under strong seismic directed to items and failure mechanisms. In short, the earthquake acceleration is converted into stored energy in node structure.

The shift holds each section in any part within the elastic region, all energy stored in the structural framework will be released at the end of the cycle in the opposite direction, the inelastic zones. When the seismic energy is too large, it will produce excessive movements that will cause a very high level curvature in the vertical and horizontal elements. This means that the rotation of the sections of the column and beams will be out of normal 
elastic range, with problems of the construction safety, particularly in phenomena such as earthquake, tornadoes and very strong crosswinds.

\section{Patent Idea}

\subsection{Brief Description of the Invention}

The principal object of the hydraulic tie rod for construction projects of the present invention as well as of the method for constructing building structures utilizing the hydraulic tie rod of the present invention is to minimize the aforesaid problems associated with the safety of construction structures in the event of natural phenomena such as earthquakes, hurricanes and very high lateral winds. According to the present invention, this can be achieved by a continuous pre-stressing (pulling) of both the building structure towards the ground and of the ground towards the structure, making these two parts one body like a sandwich. Said pre-stressing is applied by means of the mechanism of the hydraulic tie rod for construction projects. Said mechanism comprises a steel cable crossing freely in the centre the structure's vertical support elements and also the length of a drilling beneath them. Said steel cable's lower end is tied to an anchor-type mechanism that is embedded into the walls of the drilling to prevent it from being uplifted. Said steel cable's top end is tied to a hydraulic pulling mechanism, exerting a continuous uplifting force. The pulling force applied to the steel cable by means of the hydraulic mechanism and the reaction to such pulling from the fixed anchor at the other end of it generate the desired compression in the construction project.

The Reference [1] shows the mechanism of the seismic system and a seismic design method. It presents also experiments with and without the seismic patent, side by side on screen to compare the seismic protection offered by the invention. The utility of the invention has been shown experimentally.

\subsection{The Patent Idea}

We have placed on a table two columns, one column screwed on the table, and the other simply put on the table. If one shifts on the table, the unbolted column will be overthrown. The bolted column withstands the lateral loading. We do exactly the same in every column of a building to withstand more lateral earthquake loading. That is done, by simply screwing it to the ground. This pretension between the roof of the structure and the soil has been globally disclosed for the first time.

The horizontal earthquake load generates oscillation, and the result is that the upper plates shift more than the lower ones, the columns lose their eccentricity exerting a lifting force on the bases, as well as creating a twisting action in all of the nodes of the structure.

The ideal situation would be if one could construct a building framework where, during an earthquake, all the plates would shift by the same amplitude, at the ground without differing phases.

The research I have carried out resulted in just this. The method of the invention eliminates all these problems of deformation in the building construction applying pretension, through the mechanism, between the roof of the structure and the soil.

1) Comparing with existing anti seismic systems, the invention increases the strength of the structure to an earthquake over 100 per cent and reduces the cost of protection more than 50 per cent.

2) I believe that with this method, prefabricated houses can be placed in towns constructing several floors. Manufacturers and all of us will profit from this change because they are industrially produced between 30 and 50 per cent cheaper.

3) The Patent mechanism can be applied to all building projects being under construction however, it may also be placed in many existing structures, ensuring seismic protection.

Patent mechanism and method offer protection to lightweight constructions against tornadoes. It may also be used as an anchor for the support of ground slopes on highways. It ensures a strong foundation in soft ground. In addition, and all this system in a patent. There is no absolute seismic design. The invention provides the absolute seismic design. Its uniqueness makes it very marketable.

Our scientific team consists of:

1) Professor Panagiotis Karydis, Seismic Technologist-Engineer and Founder of the seismic base at National Technical University of Athens, Greece.

2) Nikos Markatos, Chemical Engineer and former Rector of The National Technical University. 
The skeleton of a building consists of the columns (vertical parts) and the girders and slabs (horizontal parts). The girders and slabs are joined at the nodes. Under normal conditions, all loading is vertical. When an earthquake occurs, additional horizontal loading is placed on the skeleton. The resultant effect of horizontal plus vertical loading puts strain on the nodes. It alters their angle from 90 degrees, creating at times acute and at other times obtuse angles. The vertical static loads equilibrate with the reaction of the ground.

The horizontal earthquake load exerts a lifting effect on the bases of the columns. In addition, due to the elasticity of the main body of the columns, the earthquake acts by shifting the heights of each plate by different amplitude and a different phase. That is, the upper plates shift more than the lower ones. The modal shifts of the skeleton are many, so many that the differing, shifting directions of the earthquake deform and destroy the skeleton.

The ideal situation would be if we could construct a building skeleton where, during an earthquake all the plates would shift by the same amplitude as the ground without differing phases. In this way the shape will be preserved and we would not have any deformation of the frame, hence no damage. The research I have carried out has resulted in the creation of an anti-seismic design for buildings which achieves exactly this result.

I have succeeded in doing this by constructing large elongated ridged columns shaped "-, +, $\Gamma$ or $T$ " to which a pulling force is applied from the roof and from the ground, applying bilateral pressure to the entire column. This force acts to prevent bilateral shifting of the columns and curvature at their bases so preventing the deformation which occurs throughout the whole structure during an earthquake.

In an earthquake, the columns lose their eccentricity and their bases are lifted, creating twisting in all of the nodes of the structure. There is a limit to the eccentricity, that is, there is a limit to the surface area of the base which is lifted by the rollover moment. To minimize the twisting of the bases, we place strong foot girders in the columns. In the large longitudinal columns (walls), due to the large moments which occur during an earthquake, it is practically impossible to prevent rotation with the classical way of construction of the foot girders.

The following result occurs with this lifting of the base in combination with the elasticity. When one column of the frame lifts one end of the beam upwards, at the same time the other column at its other end moves violently downwards. This stresses the beam and has the tendency to twist it in different directions at the two ends, deforming its body in an $\mathrm{S}$ shape. The same deformation occurs with the columns also, due to the twisting of the nodes and the differential phase shift of vertical plates. In order to prevent the lifting of the base, we clamp the base of the structure to the ground using the patented mechanism.

However, if we want to prevent the lifting of the whole columnar structure which stems from the lifting of its base as well as from the elasticity of its main body, then the best point for enforcing an opposing, balancing force is the roof. This opposing tendency on the roof must come from an external source and not applied from within the structure. This external source is the ground underneath the base. From here the external force is applied.

Underneath the base of the structure, we drill a hole into the ground and clamp it with the patented anchor. With the aid of a cable which passes freely through a pipe in the column, we transfer this force which we obtained from the ground up to the roof. At this point in the roof, we insert a stop with a screw to prevent the raising of the roof of the longitudinal columns which happens during an earthquake and deforms all the plates. In this way, we control the oscillation of whole structure. That is, the deformity which the structural failure causes. With this method, we do not see changes in the form of the structure, because it maintains the same shape it had prior to and during the earthquake.

The reaction of the mechanism to the raising of the roof of the longitudinal column and the opposing reaction of the at the bottom part of the base, divert the lateral load of the earthquake in the strong vertical section. With this diversion of the lateral load of the earthquake to the vertical columns, the twisting of the nodes is abolished because the lateral loadings of the earthquake are 100 per cent borne along the length of the columns, so it is impossible for them to twist in their main sections.

In the experiments I have carried out in actual scale earthquake acceleration of "1.77" and amplitude over " 0.11 " in a two story building model to scale " $1: 7.14$ ", the difference in the model with and without the patented mechanism can clearly be seen [1] experiments,

1) With the seismic system [2];

2) Without the seismic system [3].

I'll give some theoretical elements to do and check yourself I did calculations [2]. The model I performs a simple harmonic oscillation along the "axis $(X)$ " on which commutes (ignoring the vertical movement is small). 
This reciprocating motion generated by the circular movement of the end of the piston which is attached the bearing pin is. The radius of this circle is " $0.11 \mathrm{~m}$ " and this is the "amplitude $A$ ". Thus my make model path " $2 A=$ $0.22 \mathrm{~m}$ ", i.e. go from one end point to another in each half turn of fire.

A complete oscillation but means the pin is to make a full turn, i.e. to return the model to the extreme position from where he started. So, if we say that it started from the end should be restored at the end. Makes therefore overall route that went " 0.22 " and " 0.22 " that turned as " $4 A=0.44 \mathrm{~m}$ ". So if you stand by the side of the machine and measure routes, each approach to the machine is a complete path and thus a turn. These speed counting, and the corresponding time in sec. The "frequency (Hz)" is the "Equation (1), fraction: $n=$ number of such full path/same time". "The period of oscillation T", i.e. the time of a full stroke " $0.44 \mathrm{~m}$ " is " $T=1 / \mathrm{n}$ sec". In a full turn of fire, we once maximum positive speed in one direction and once the maximum negative in the other. Us of course we are interested in the absolute values that are the same. The same happens with the acceleration, but has maximum absolute value when the speed is zero, the ends of the paths.

Maximum speed and maximum acceleration calculated from the "angular velocity $\omega$ " is "Equation (2), $\omega=$ $2 \pi / T$ ". So, Equation (3) is, "maximum speed $U$ : $\operatorname{maxv}=\omega * A=0.11 \mathrm{~m} / \mathrm{sec",} \mathrm{"Equation} \mathrm{(4)} \mathrm{is} \mathrm{maximum} \mathrm{acce-}$ leration $a$ : $\max \alpha=\omega^{2} * A=0.11 \mathrm{~m} / \mathrm{sec}^{2}$ ". These maximum sizes made instantaneously. If we take the average acceleration, either positive or negative, then we think that the speed went from zero to its maximum at time " $T / 4$ ". So the average speed is approximately "Equation (5) is a $=\operatorname{maxv} /(T / 4)=4 * \operatorname{maxv} / T=4 * 0.11 . \omega^{2} / T$ in $\mathrm{m} / \mathrm{sec}^{2}$ ". This of course is not true, because at the time " $T / 4$ " the " $a$ " is greater (not entangle you with cosines and sines).

In both instances, however, to find the acceleration in g, we must divide the accelerations are " $\mathrm{m} / \mathrm{sec}^{2}$ " the Earth accelerating mass is " $9.81 \mathrm{~m} / \mathrm{sec}$ " to say that we have achieved so many acceleration g. I think I was detailed. What we do in practice and what other factors are taken into account, is a challenge;

\subsection{Analytical Results of the Experiment}

From 2.45 minutes to 2.50 minutes in 5 seconds makes 10 complete turns [2].

That is "40 full turns in $20 \mathrm{sec",}$

1) So amplitude $A=0.11 \mathrm{~m}$,

2) The Frequency (Hz) "Equation (1) is $n=$ number of such full path/corresponding time”.

So $40 / 20=2 \mathrm{~Hz}$,

3) The fundamental period of the oscillation period T, i.e., the time of a full stroke " $0.44 \mathrm{~m}$ ", the "Equation (6) is $\mathrm{T}=1 / \mathrm{n} \mathrm{sec}$ ". So " $1 / 2=0.5 \mathrm{sec}$ ",

4) Angular velocity is "Equation (2), $\omega=2 \pi / T$ ". So " $2 \times 3.14 / 0.5=12.56$ ",

5) Max speed U "Equation (3) is $\max v=\omega * A=0.11 \mathrm{~m} / \mathrm{sec".} \mathrm{So} \mathrm{"} 12.56 \times 0.11=1.3816 \mathrm{~m} / \mathrm{sec}$ "

6) Maximum acceleration $\alpha$ Equation (4) is $\max \alpha=\omega^{2} * A=0.11 \mathrm{~m} / \mathrm{sec}^{2}$ ” $=0.11 \mathrm{~m} / \mathrm{sec}^{2}$.

So " $12.56 \times 12.56 \times 0.11=17.352896$ ",

7) “Acceleration in g “Equation (7) is max $/$ Earth accelerating mass = 17,352,896/9, $81=1.77 \mathrm{~g}$ ”.

Excludes the vertical acceleration.

That model is a scale that raises accelerate too much more than " $1.77 \mathrm{~g}$ " but measured differently than that I counted, and out of math that I do not know. (Which relate mass and acceleration and earn some scales) these types know their test labs.

This acceleration is acceleration took off real natural earthquake, on a small scale model of 1 to 7.14 . This told me the professor. The largest earthquake ever in the world, was $2.99 \mathrm{~g}$. The strongest structures in Greece built to withstand $0.36 \mathrm{~g}$.

To My model was tested at $1.77 \mathrm{~g}$ and was not hurt, so I do not know when it fails. In Greece the largest earthquake that was reached in the $1 \mathrm{~g}$ acceleration. Correlation with the Mercalli scale [4].

\subsection{There Are Many Design Methods}

The first method.

My friends we can try this method includes horizontal seismic insulation and one or more embedded rigid central fiery. An elastic skeleton of a building with horizontal seismic insulation and therein one or more independent rigid bodies [5].

The second method. Design with elongated rigid columns. 
The horizontal earthquake load exerts a lifting effect on the bases of the columns. In addition, due to the elasticity of the main body of the columns, the earthquake acts by shifting the heights of each plate by different amplitude and a different phase. That is, the upper plates shift more than the lower ones. The modal shifts of the skeleton are many, so many that the differing, shifting directions of the earthquake deform and destroy the skeleton.

The ideal situation would be if we could construct a building skeleton where, during an earthquake all the plates would shift by the same amplitude as the ground without differing phases. In this way the shape will be preserved and we would not have any deformation of the frame, hence no damage.

The research I have carried out has resulted in the creation of an anti-seismic design for buildings which achieves exactly this result. I have succeeded in doing this by constructing large elongated ridged columns shaped "-,,$+ \Gamma$ or $\mathrm{T}$ " to which a pulling force is applied from the roof and from the ground, applying bilateral pressure to the entire column. This force acts to prevent bilateral shifting of the columns and curvature at their bases so preventing the deformation which occurs throughout the whole structure during an earthquake.

In an earthquake, the columns lose their eccentricity and their bases are lifted, creating twisting in all of the nodes of the structure. There is a limit to the eccentricity, that is, there is a limit to the surface area of the base which is lifted by the rollover moment. To minimize the twisting of the bases, we place strong foot girders in the columns.

In the large longitudinal columns (walls), due to the large moments which occur during an earthquake, it is practically impossible to prevent rotation with the classical way of construction of the foot girders.

The following result occurs with this lifting of the base in combination with the elasticity. When one column of the frame lifts one end of the beam upwards, at the same time the other column at its other end moves violently downwards.

This stresses the beam and has the tendency to twist it in different directions at the two ends, deforming its body in an S shape. The same deformation occurs with the columns also, due to the twisting of the nodes and the differential phase shift of vertical plates. In order to prevent the lifting of the base, we clamp the base of the structure to the ground using the patented mechanism.

However, if we want to prevent the lifting of the whole columnar structure which stems from the lifting of its base as well as from the elasticity of its main body, then the best point for enforcing an opposing, balancing force is the roof. This opposing tendency on the roof must come from an external source and not applied from within the structure. This external source is the ground underneath the base. From here the external force is applied.

Underneath the base of the structure, we drill a hole into the ground and clamp it with the patented anchor. With the aid of a cable which passes freely through a pipe in the column, we transfer this force which we obtained from the ground up to the roof. At this point in the roof, we insert a stop with a screw to prevent the raising of the roof of the longitudinal columns which happens during an earthquake and deforms all the plates.

In this way, we control the oscillation of whole structure. That is, the deformity which the structural failure causes. With this method, we do not see changes in the form of the structure, because it maintains the same shape it had prior to and during the earthquake.

The reaction of the mechanism to the raising of the roof of the longitudinal column and the opposing reaction of the at the bottom part of the base, divert the lateral load of the earthquake into the strong vertical section.

With this diversion of the lateral load of the earthquake to the vertical columns, the twisting of the nodes is abolished because the lateral loadings of the earthquake are $100 \%$ borne along the length of the columns, so it is impossible for them to twist in their main sections.

And the third method, the prestressed columns do not have ductility, and cannot absorb energy.

What I do to solve this problem. Simply, I do not apply pretension between the roof and drilling. What am I doing?

First apply pretension between the level of the foundation base (ground) and the anchor mechanism which is in the depths of the hole of drilling. The pretension is twice than it is the axial loads I want to receive the tendon in an earthquake. The initial prestressing applied to achieve very strong adhesion (Clamping) of the anchor into the walls of the borehole. Then fill the hole drilling with Concrete.

After uniting the tendon that extends from the borehole, with a nut, to lengthen until the roof. We take care of the tendon to pass through a plastic tube free, so to avoid guilds (adhesion) with this concrete. On the roof, inserted between the tendon and the roof a spring which simply tighten with a screw. Do not apply any other 
second pretension. The spring on the roof leaves the column to oscillate inside the elastic range while applying seismic damping because it prevents the rise of the roof of the long column. But stop the column to pass on inelastic failure region.

\subsection{Conclusions}

The construction framework with ultimate anti-seismic system makes strong foundation in earthquakes, solarwind and generally in energy sector structural systems, may limit the impact of tornadoes effects into lightweight constructions, may give more stable behavior for Towers buildings and may suggest to fix existing or under design construction like as bridges.

The paper concludes that the patent is a vibration control system, with references [6]-[8], with innovation hydraulic tie rod and brings potential decrease of risk management in node structural contraction. However, influence independently from steel beam strengthens the compatibility of the overall behavior in seismic risk.

I suggest that to build up an institution for the specific scientific metrics and consultation for the community with the guidelines, also big data analytics, perhaps improve the choice to support the priorities and implementation areas in the world with highest risk catastrophe.

\section{Acknowledgements}

I would like to express our hearty thanks to Molson A.F.A., MHSA, Argyroupoli, Attica, Greece, for the valuable bucking up support and encouragement on me for the article.

\section{References}

[1] Lymperis, I.N. (2014) Anti-Seismic System. https://www.youtube.com/watch?v=zhkUlxC6IK4

[2] Lymperis, I.N. (2014) The Ultimate Earthquake Experiment for System Construction. https://www.youtube.com/watch?v=RoM5pEy7n9Q

[3] Lymperis, I.N. (2014) The Experiment without Anti-Seismic Control System Bring Overall Construction (100\%) Collapse. https://www.youtube.com/watch?v=l-X4tF9C7SE

[4] Wikipedia, The Free Encyclopedia (2015) Peak Ground Acceleration. https://en.wikipedia.org/w/index.php?title=Peak_ground_acceleration\&oldid=671564807

[5] Lymperis, I.N. (2014) Anti-Earthquake Systems.https://www.youtube.com/watch?v=IO6MxxH0lMU

[6] Lymperis, I.N. (2009) Hydraulic Tie Rod for Construction Projects. The Patent Cooperation Treaty (PCT) Pub. No.: $\mathrm{WO} / 2010 / 097642$.

[7] Lymperis, I.N. (2009) Tie Rod for Structural Projects. The Patent Cooperation Treaty (PCT) Pub. No.: WO/2009/101454.

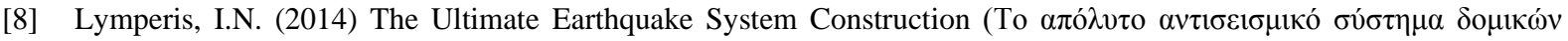

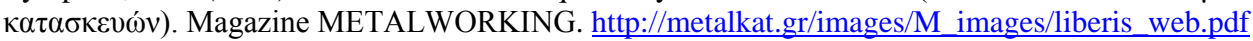

